\title{
Experimental Constraints on Supersymmetric Theories
}

\author{
J.P. Leveille
}

Randall Lab of Physics, University of Michigan, Ann Arbor, MI 48109

\section{ABSTRACT}

We extend the analyses of Fayet, and Fayet and Farrar, of experimental searches for gluinos, the supersymmetric partners of gluons. Because of their large production cross sections, present data appears to exclude gluino masses below $3.5-6 \mathrm{GeV} / \mathrm{c}^{2}$ and may be more restrictive. Since gluinos remain very light in many models, they will either be detected soon or many supersymmetric theories will be excluded.

\section{INTRODUCTION}

In this talk we would like to address the question: Is supersymmetry a symmetry of nature? We will conclude that present experimental data places very strong constraints on possible supersymmetric models. To obtain these constraints we restrict ourselves to the study of gluinos, the supersymmetric partners of the gluons. For clarity, a brief introduction to supersymmetry and a user's guide to the plethora of supersymmetric particles is first given. After explaining why the gluinos are a sensitive probe of supersymmetry, the results will be presented. The last part of the talk will explain how these results are arrived at, by studying the properties of gluinos and the constraints from present data, and data soon to be obtained.

The details of the analysis are contained in a paper by G.L. Kane and the author, ["Experimental Constraints on Gluino Masses and Supersymmetric Theories", Michigan preprint UM HE 81-68] from which large sections of this write up are plagiarized. We refer the interested reader to that paper for more details and the necessary references.

\section{SUPERSYMMETRY}

Supersymmetry (SS) is a symmetry between bosons and fermions. Recall what we mean by isospin: the proton and the neutron are different isospin states, related by isospin generators $T$, i.e. $\mathrm{T}^{+} \mid$neutron $\rangle=\mid$protón $\rangle$.

In SS, there are fermionic generators $Q_{\alpha}$, which connect fermion states with boson states: $Q_{\alpha} \mid$ boson $\rangle=\mid$ fermion $\rangle$. These fermionic generators also commute with the Hamiltonian, $\left[H, Q_{\alpha}\right]=0$. Consequently, there must be a degeneracy in the mass spectrum of boson and fermions if SS is a symmetry of the world! This is clearly not the case: there are no scalars degenerate in mass with the electron or the muon for example. Rather than throw out the whole concept, today's theoretical prejudices lead us to believe that supersymmetry could be a broken symmetry of nature. Since it 
necessarily must be broken, one may well ask what is the use of SS? There are great hopes that gravity could be incorporated more easily into a supersymmetric theory. However in our days of unified theories, the real appeal of supersymmetric models is that they would: (a) naturally incorporate scalars (bosons) into the theory, (b) because the bosons and the fermions are connected by the supersymmetry keeping the fermions massless would also lead to massless bosons. This latter point is crucial. The model one envisages is a GUT model together with a supersymmetry SS. The supersymmetry commutes with the grand unified group. When the grand unified group breaks, at MGUT, the supersymmetry remains unbroken, e.g. SS $\times$ GUT + SS $\times$ SU(3) $\times S U(2) \times U(1)$. Because the broken theory is still supersymmetric we can now keep the fermions and hence the Higgs bosons associated with them massless in a naturat way, for example, using a chirat symmetry. When the supersymmetry finally breaks at $\Lambda_{S} S^{\sim} M_{W}$, the ordinary Higgs mechanism gives masses to bosons - $M_{W}$. So although this model of the world would not explain why $M_{G U T} \gg M_{W}$, at least the introduction of SS makes the theory natural: we do not force the scalar masses to vanish, a symmetry does it!

The models of supersymmetry which exist nowadays, always have a large number of particles. To orient the reader we now give a guide to the particles likely to exist in a model such as the one outlined above. First of all each conventional particle has a supersymmetric partner differing in spin by half a unit. All the internal quantum numbers of the supersymmetric partner are the same as those of the conventional particle. In the list below we only include the electric charge $Q$, but the same is true for color, isospin etc...

\begin{tabular}{|c|c|c|c|c|c|c|}
\hline \multicolumn{2}{|c|}{ CONVENTIONAL } & \multirow{2}{*}{$\frac{\text { SPIN }}{1 / 2}$} & \multirow{2}{*}{$\begin{array}{l}\underline{Q} \\
-1\end{array}$} & \multicolumn{2}{|l|}{ SS PARTNER } & \multirow{2}{*}{$\frac{\text { SPIN }}{0}$} \\
\hline Leptons: & $e, \mu, \tau, \ldots$ & & & Scalar leptons: & $\phi_{e}, \phi_{\mu}, \phi_{\tau}, \ldots$ & \\
\hline Ouarkc. & $u, c \ldots$ & $1 / 2$ & $2 / 3$ & Scalar & $\phi_{u}, \phi_{C}$ & 0 \\
\hline Qudrks: & $d, s, b, \ldots$ & $1 / 2$ & $-1 / 3$ & rK S: & $\phi_{d}, \phi_{s}, \phi_{b} \ldots$ & 0 \\
\hline gluons: & $g$ & 1 & 0 & gluinos & $\widetilde{g}$ & $1 / 2$ \\
\hline $\begin{array}{l}\text { Photon: } \\
\text { - } \\
\text { - }\end{array}$ & $\gamma$ & 1 & 0 & photino & $\tilde{Y}$ & $1 / 2$ \\
\hline$\dot{z}$ & & 1 & 0 & Zino & $\tilde{z}$ & $1 / 2$ \\
\hline NONE & & & 0 & GOLDSTINO & G & $1 / 2$ \\
\hline
\end{tabular}

Note that there is one particle the goldstino, G, which does not have a conventional partner. This particle is a Goldstone fermion, which appears when a global SS is spontaneously broken. It has the same quantum numbers as the vacuum, but has spin 1/2. 
If the SS was a local symmetry, then the goldstino would become part of a spin $3 / 2$ particle, in much the same way as Goldstone bosons become the zero helicity states of the gauge bosons in the standard model of weak interactions.

We will now explain why gluinos are interesting and can potentially constrain SS models. Indeed it turns out that in the simplest SS models of strong, weak and electromagnetic interactions which can be unified, it is very difficult to give the gluinos a large mass. If we consider the collection of existing models which are not ruled out by other diseases, we find very conservatively that the gluino mass $\tilde{m}$ is always bounded above by $2 \mathrm{GeV}$ : $\tilde{m}<2 \mathrm{GeV}$. Why? We11, first there are no bare mass terms because of the supersymmetry. Otherwise gluons would have a mass term. When one breaks the SS, one does not want to break color, so no mass term can be generated for the gluinos (it would require a VEV for a colored scalar, which would break color). Hence the gluino masses must be generated radiatively. One loop diagrams actually vanish because of a spurious (accidental) symmetry, called R-invariance,

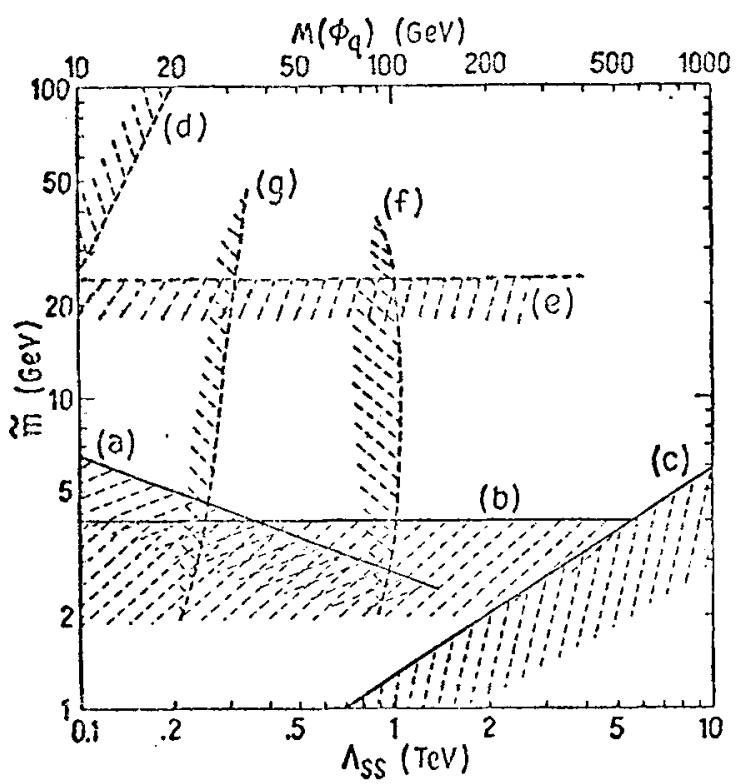
which crept up in the original Lagrangian without one wanting to put it there. So the masses only arise at two loop level, hence their smaliness.

So we expect the gluinos to be "light" colored fermions, which will therefore be produced copiously at hadron machines.

Failure to detect them constrains the models. Detecting them also constrains the models (amongst other things). The results of our analysis are presented in Figure 1. There we

Fig. 1. Excluded region in the $\tilde{m}-\Lambda S S$ plane. Solid curves are from present data. Dashed curves are attainable limits from future experiments. (a) Valid if $\tilde{g} g G$ vertex is present. (b) From $\tilde{g}+q \bar{q} \tilde{\gamma}$ with subsequent $\widetilde{\gamma}$ interactions in a beam dump detector. This curve is always present. We have assumed $M_{\phi q}=M_{W} / 2$. (c) Gives upper limit on $\Lambda$ SS (lower scale) or $M_{\phi p}$ (upper scale) from the requirement that $\mathrm{g}$ lifetime not be too fong. (d) Upper limit on $\tilde{m}$ from double goldstino production at Isabelle. (e) The region below this line would be excluded by a failure to detect gluino production by an SPS detector with $f=1029 / \mathrm{cm}^{2}$. (f) The region to the left is excluded if 100 events of $G+\tilde{g}$ production are not detected at I SABELLE. (g) Same as ( $f$ ) for FNAL collider. 
show the regions of the gluino mass $\tilde{m}$ - scale of supersymmetry breaking $\Lambda$ SS plane excluded by present data (shaded regions below solid curves) and accessible to future experiments (shaded regions below dashed curves). Note that present experiments already rule out gluinos lighter than $4 \mathrm{GeV}$. This alone signals the demise of existing models.

\section{RESULTS}

We now indicate how the results of Fig. 1 are arrived at. The basic strategy will be to calculate the production cross-sections for gluinos as a function of their mass $\widetilde{m}$. After specifying their decay modes, experiments will set lower limits on their masses.

(1) Gluino couplings: Since gluinos are the partners of gluons they witl have the interactions shown in Fig. 2.a. The coupling at each vertex is the standard QCD coupling $g$. In addition, for a broken SS a coupling to the goldstino is introduced, as in $\mathrm{Fig}$. 2b. Gauge invariance requires a magnetic type coupling, $h \bar{u}_{G} \sigma^{\mu u_{u}}{ }_{F}^{a}{ }_{\mu \nu}$ where $u_{G}$ and $u_{g}^{a}$ are spinors for a goldstino and gluino of color a, respectively, and $F_{\mu \nu}^{a}$ the gluon field strength. The coupling strength $h$ is fixed by supercurrent algebra. Indeed taking the matrix element of the supercurrent $S_{\mu}$ between a gluino and a gluon, including the goldstino pole term and requiring zero divergence, yields $h=\tilde{m} / 2 \Lambda_{S S}^{2}$ where $\tilde{i}$ is the gluino current algebra mass and $\Lambda_{2} S$ sets the scale of SS breaking, defined by $\left\langle 0\left|S_{\mu}\right| G\right\rangle=\Lambda_{S S}^{2} \gamma_{\mu} u_{G}$.

Some of our results only require the interactions of Fig. $2 \mathrm{a}$, for production of gluinos via gluons. They hold in any theory

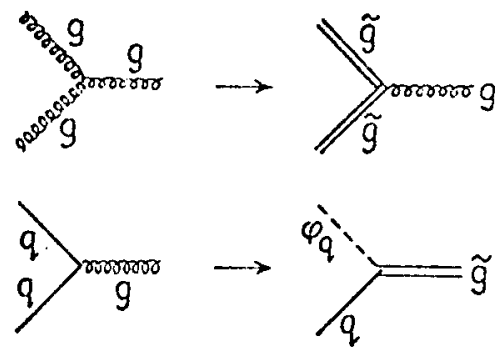

(a)

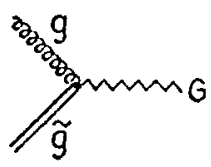

(b)

Fig. 2. Gluino couplings in supersymmetric theories. We represent gluons by $g$, gluinos by $\widetilde{g}$, goldstinos by $G$, quarks by $q$, scalar partners of quarks by $\phi_{q}$, and the photino by $\tilde{\gamma}$. The vertices of (a) will be present in every supersymmetric theory when gluinos carry color. The vertex of (b) is present in global supersymmetric theories. 
where the gluinos are color octets. In local SS the Goldstino may become the helicity $1 / 2$ part of a spin $3 / 2$ state so our results involving Goldstinos may not directly hold. However, since the gluinos will have to decay (see below), essentially equivalent results will be valid.

(2) Gluino Lifetimes and Interactions: In a spontaneously broken globaT SS, the decay of the gluino proceeds dominantly via the vertex in Fig. 2b. We obtain the lifetime

$$
\tilde{\tau}_{G}=\frac{33 \times 10^{-15}}{h^{2} \Lambda_{S S}^{4}}\left(\frac{\Lambda_{S S}}{M_{Z}}\right)^{4}\left(\frac{1 \mathrm{GeV}}{\tilde{m}}\right)^{3} \text { sec. }
$$

Since current algebra arguments yield $h \simeq \tilde{m} / 2 \Lambda^{2} S$, this becomes

$$
\tilde{\tau}_{G} \simeq 1.1 \times 10^{-15}\left(\frac{\Lambda_{S S}}{M_{Z}-}\right)^{4}(\underbrace{1 ~ G e V}_{\tilde{m}})^{5}(\mathrm{sec})
$$

If observations imply $\tilde{\mathrm{m}}>3 \mathrm{GeV}$, and $\Lambda \mathrm{SS}<1 \mathrm{TeV}$ for the cases of interest, then $\widetilde{\tau}<.7 \times 10^{-13} \mathrm{sec}$. If $\widetilde{g}$ is produced with $\gamma=20$, it will travel typically .4 mm. On the other hand, if $\tilde{m} \simeq 1 \mathrm{GeV}$, and $\Lambda_{S S}=1 \mathrm{TeV}$, it goes $3^{6}$ times further, typically 0.30 meters with, of course, some going over a meter. Note that Eq. (2) provides an interesting upper limit on $\Lambda S S$ for a given $\tilde{m}$ (See Fig. 4). If data excludes production of a gluino which travels more than about 10 $\mathrm{cm}$. (see below), then any theory must satisfy $\Lambda \mathrm{SS} / \mathrm{m}^{1.5} \lesssim 1000$, with $\Lambda$ SS and $\tilde{m}$ in GeV units.

If the $\widetilde{g} g$ vertex is suppressed or absent as perhaps could occur in a local SS, the gluino will decay via a virtual scalar quark to a quark-antiquark pair and a photino $(\tilde{\gamma})$ (provided that the gluino is heavier than the photino).

For the mode $\widetilde{g} \rightarrow q q \widetilde{\gamma}$, the lifetime is

$$
\widetilde{\tau}_{\widetilde{\gamma}}=0.8 \times 10^{-6}\left(m_{\mu} / \tilde{m}\right)^{5}\left(M_{\phi} / M_{W}\right)^{4} \text { sec. }
$$

$M_{\phi}$ is the lightest scalar quark mass associated with quarks lighter than the gluino. By comparison, the $\widetilde{\gamma}$ mode dominates if $M_{\phi}<0.09$ $\Lambda S S$; if $M_{\phi}=M_{W} / 2$, the photino mode dominates for $\Lambda_{S S} \geq 400 \mathrm{GeV}$ (see Fig. 1).

Thus we expect that experiments sensitive to neutral hadrons that can travel centimeters or meters will give a lower limit on the gluino mass. When a gluino is produced it will be shielded to make a color singlet hadron. Most probably the gluino will bind with a gluon, because of the octet binding forces, though sometimes

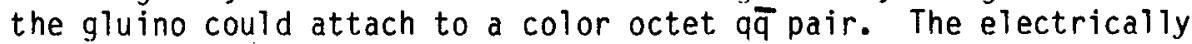
neutral, color singlet, hadron will interact like a normal hadron, with a total interaction probability like that of a kaon or a $D^{\circ}$, with oTOT - few mb. As observed by Fayet and Farrar, and as we will reaffirm below, any objects produced with several ub cross 
sections, and having such lifetimes and interactions, would probably have been observed.

(3) Gluino and Goldstino Production:

Once the smaTt mass range if $\lesssim I G e V$ is excluded by the absence of long-lived, electricality neutral, strongly interacting hadrons, we can reliably use perturbative QCD to calculate (lower limits on) the production cross sections, and these are very large for color

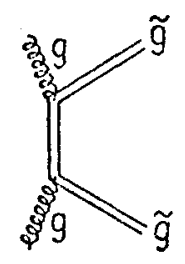

(a)

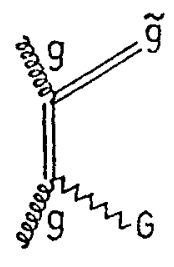

(b)

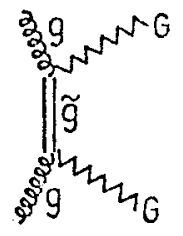

(c) octet gluinos. Further, in any theory where there is a g̃gG coupling, the double or single direct goldstino production cross (Fig. 3b-c) sections increase with $\tilde{\mathrm{m}}$ and the absence

Fig. 3. Production mechanism for gluinos and goldstinos. (a) is present in any theory where gluinos carry color. It gives the cross sections of Fig. 4 for color octet gluinos. (b) and (c) are present in globally supersymmetric theories and give upper 1 imits of Fig. 1.

of experimental detection of such events will give an upper limit on $\tilde{\mathrm{m}}$.

We show the gluino pair-production mechanisms for $p p$ and $p \bar{p}$ collisions in Fig. 3. Figure 4 shows the production cross sections vs. the gluino mass $\tilde{m}$ for a number of beam energies. Since the curves fall rapidly, a small error in estimating the cross section limit has little effect on the associated mass i imit. Once again these are expected to be conservative lower limits since production of $c \bar{c}$ and $b \bar{b}$ is larger than the perturbative prediction.

It should be emphasized that the cross sections are quite large. The actual calculation includes not only the diagram of Fig. 3a, but the crossed graph, the direct gluon pole term, and the production via quarks, $q \bar{q}+g+\widetilde{g} \tilde{g}$. In the region of interest the subprocess shown is the largest one in the Feynman gauge, and to understand the size of the cross section we can compare it to $q \bar{q}$ production. With generators $F^{a}$ in the octet representation and $\lambda^{\mathrm{a}} / 2$ in the fundamental representation, we have for equal kinematics, and infinite energy,

$$
\frac{\sigma(g g+\tilde{g} \tilde{g})}{\sigma(g g \rightarrow q \bar{q})} \approx \frac{\operatorname{Tr} F^{a} F^{a} F^{b} F^{b}}{\operatorname{Tr} \lambda^{a} \lambda^{a} \lambda^{b} \lambda^{b} / 16}=13.5 \text {. }
$$




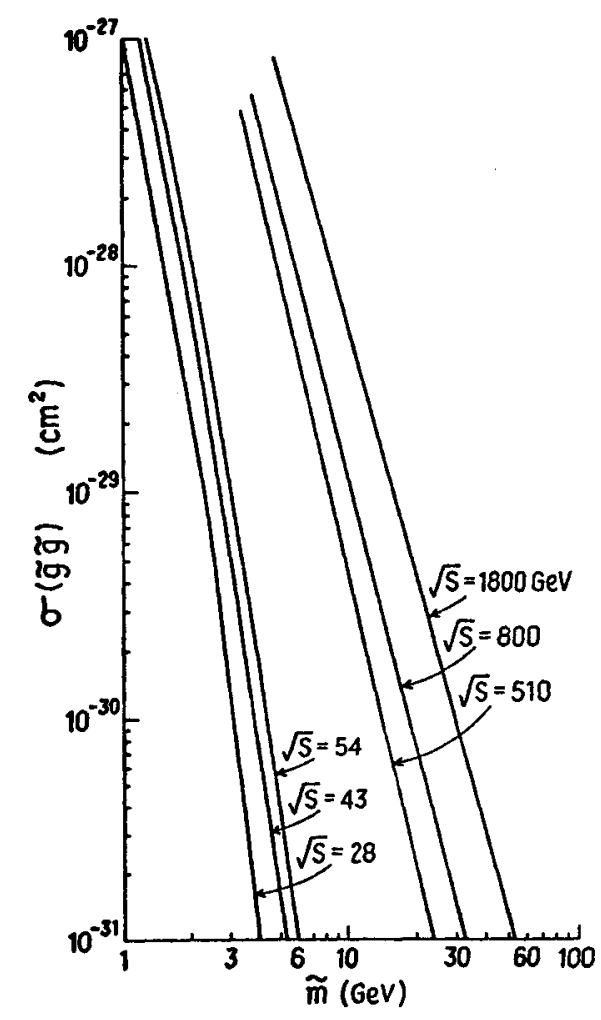

For the actual calculation the gluino pair cross section varies from 16-20 times the cross section for production of a pair of quarks of the same mass. (4) Retroactive Analys is of Data: It is obviousty difficult to analyze existing experiments to see what limits they put on gluino masses. It has even been suggested that experimenters only find what they are looking for. We will abstract from past data some estimates on what might have been seen; our results are summarized in Fig. 1. We want to emphasize that they are only estimates, and should not be taken as firm limits until experimenters have analyzed their own data with a full knowledge of backgrounds, cuts, etc. Experiments in progress can set significant ty better Timits than we obtain if they are anatyzed with gTuino (or gotdstino) detection in mind, and experiment s at SPS, ISABELLE or FNAL can go to very high masses.

Fig. 4. Gluino production cross sections computed from Fig. $3 a$, including scaling violations, as parameterized by Baier et al. for several values of $\sqrt{\mathrm{s}}$ (in GeV).

(a) Sriall Gluino Masses and Longer Lifetimes: As discussed above, if III is of the order of I GeV the lifetime is fairly long. Fayet and Farrar have al ready argued that this is not allowed by data, and we agree. The case can be made very strong. For small $\widetilde{m}$, while perturbative calculations are not reliable, the production cross section will not be smaller than that of Fig. 4, so o $21 \mathrm{mb}$. Produced gluinos will be shielded by gluons or qव octet pairs, so an electrically neutral hadron will be produced, travel a distance from millimeters to meters, and decay into an even number of 
charged hadrons (often four or more hadrons) which do not point back to the production vertex. There is missing energy because of the Goldstino (or photino) but no charged leptons. The shielded gluino will interact with a total cross section in the mb range. The experiment of Gustafson et al can put limits of order $10^{-32} \mathrm{~cm}^{2}$ on any neutral object produced in appropriate regions of $p_{T}$ and $X_{F}$ which goes several meters and then interacts with a millibarn cross section. Experiments in hyperon beams may be able to put limits of order $10^{-3}-10^{-4}$ times the $\Lambda$ cross section on objects which go a few meters and decay into an even number of charged prongs which do not point back to the production vertex. In hydrogen bubble chambers there are strong restrictions on events which would give a visible gap and an even number of prongs (neutrons give a recoil proton and an odd number of prongs). Altogether, we think it is convincing that objects with the properties of light gluinos are not produced with cross sections of even a few $\mu \mathrm{b}$, so $\tilde{m} \geq 2-3 \mathrm{GeV}$. If $\sigma<1 / 2$ $\mu \mathrm{b}$, then $\mathrm{m} 23.5 \mathrm{GeV}$. We assume fixed target $\mathrm{pp}$ collisions with $\sqrt{\mathrm{s}}$ $\simeq 28 \mathrm{Gev}$ for these numbers; they vary a little for other energies or beams.

(b) Beam Dump Experiments: Once the mass is as large as estabtished in (a) above, most gluinos decay within a few $\mathrm{cm}$, and either beam dump or missing energy detectors will be most restrictive. In beam dump experiments the goldstino will interact in the detector, giving no charged lepton and thus candidate neutral current (NC) events. Recent experiments looking for axions quote an upper limit $(2 \sigma) \cdot \sigma_{j n t}<2 \times 10^{-67} \mathrm{~cm}^{4}$ where $\sigma$ is the production cross-section for the gluino in our case and oint the interaction cross-section for the goldstino. Assuming that the goldstino interaction is like a charged current neutrino interaction, and an average energy of $60 \mathrm{GeV}$ for the goldstino (a typical $v$ energy in such an experiment), we find again that $\sigma \lesssim 1 / 2$ $\mu \mathrm{b}$ or equivalently $\tilde{\mathrm{m}} \geq 3.5 \mathrm{GeV}$.

For some ranges of $\tilde{m}$ and $\Lambda$ SS this result can be considerably strengthened by further data analysis. First, in any theory with a $\tilde{g} g G$ vertex the goldstino interaction will be much larger than the $v$ charged cross-section. Indeed the Goldstino can interact with protons in the detector by fusing with a constituent gluon. Using $60 \mathrm{GeV}$ for a mean goldstino energy and $\Lambda_{S S}=300 \mathrm{GeV}$, we find that the goldstino interaction cross section $\sigma_{G}$ is larger than $\sigma_{\nu}$ in the range $1 \mathrm{GeV} \leq \tilde{i}$ $\lesssim 6 \mathrm{GeV}$ and the Goldstino interaction cross-section is increased by a factor of 4-6 over the contributions considered previously. This strengthens the previous $1 \mathrm{imits}$ and pushes $\tilde{m}$ to about $4.5 \mathrm{GeV}$. Second, a $\vee N C$ event has large missing PT for the hadrons, and a spectrum of visible hadron energy ( $E_{v i s}$ ) which peaks at low Evis and does not have a long tail. A goldstino induced event, on the other hand, will have considerably larger $E_{v i s}$ (thus it could not account for any extra events at smal1 $E_{y j}$ ) and much smaller ( $\mathrm{pT} / \mathrm{PL}_{\mathrm{L}}$ )had. Cuts on these variables could eliminate most $v$ NC candidates and allow a small goldstino signal to be found, or give a limit well below $1 \mu b$. 
The photino interaction cross section is dominated by the process $\widetilde{\gamma}+q+q+\widetilde{g}$, with a scalar quark exchanged, as discussed by Fayet. This gives a cross section

$$
\sigma_{\text {int }}=1.2 \times 10^{-38} E\left(M_{W} / M_{\phi}\right)^{4} \sum_{q} \int_{\tilde{m}^{2} / s}^{1} x q(x)\left(1-m^{2} / x s\right)^{3} d x e_{q}^{2}\left(\mathrm{~cm}^{2}\right)
$$

with $\mathrm{E}$ in $\mathrm{GeV}$. We assume that the lightest scalar quark has $M_{\phi}=$ $M_{W} / 2$. Then combining this with the beam dump limit gives curve (b) of Fig. 1, drawn for fixed $M_{\phi}$ and $\Lambda S S$ (in a particular theory, $M_{\phi}$ may depend on $\Lambda_{S S}$ ). Even if the goldstino is not present the photino decay together with the beam dump data already provides a stringent lower limit on $\mathrm{m}$.

(c) Missing Energy and pT Experiments: The most powerfut 1 imits wi T1 come from experiments, at Tevatron and collider energies, which constrain missing energy and momentum as well as possible. Again, we emphasize SS theories with a ggG vertex, but our remarks apply also to theories without such a vertex so far as a lower limit on $\tilde{m}$ is concerned. The upper limit on $\tilde{m}$ depends crucially on such a vertex.

Consider an experiment at the ISR pp collider with a typical integrated luminosity of $10^{37} / \mathrm{cm}^{2}$. Then if $\sigma>10^{-33} \mathrm{~cm}^{2}$ it had $10^{4}$ gluino pairs produced. This corresponds to $\tilde{\mathrm{m}} 210 \mathrm{GeV}$ if gluinos were not found. Similarly, consider $\int \mathrm{fdt}=10^{35} / \mathrm{cm}^{2}$ at the SPS collider. Then $10^{4}$ events correspond to $\sigma=10^{-31} \mathrm{~cm}^{2}$, or $\mathrm{m} \geq 24$ GeV!

Could such events have been seen al ready? Their signature is fairly dramatic. The gluinos are produced in the central region, and decay, say, via $\tilde{g}+g G$. The gluon gives a hadronic jet, so there is a pair of acoplanar jets, plus a lot of missing transverse energy and momentum, and no prompt charged leptons. Typically, about $25 \%$ of the energy will go into the central collision, so $10-15 \%$ of the total energy and about half of the central energy will be missing. Certainly $10^{4}$ such events could be found in ISR or SPS experiments specifically looking for them in the near future.

(d) Upper Limits: Since the cross sections for double goldstino production grow as $\tilde{m}$, they give upper limits on $\tilde{m}$ or lower limits on $\Lambda$ SS if a signal is not found. The signature for goldstino pair production is an event with an interaction and beam jets but essentially no central region energy. The goldstino-gluino production is easier to see as $\widetilde{g}+\mathrm{gG}$ giving one jet (or $\widetilde{g}+q \bar{q} \hat{\gamma}$ ), with no particles detected in the opposite direction. Neither type of event has prompt charged leptons. These give the future curves $d, f, g$ of $F i g .1$.

\section{Conclusions}

Since gluinos tend to be lighter than other supersymmetric partners, and are produced with large cross sections, they should 
be considered as the prime hope in deciding experimentally whether nature is supersymmetric. We think, conservatively, that gluinos would probably have been detected if their masses were in the excluded region of Fig. 1; basically, $m>4 \mathrm{GeV}$. Analysis of existing data by experimenters, and experiments in progress, can strengthen these limits considerably if gluinos are not detected. Since gluino properties depend on the scale of SS breaking and on scalar quark masses, interesting upper/lower limits on all of these are implied by upper limits on lifetimes of long-lived neutral hadrons and on production cross sections.

\section{ACKNOWLEDGMENTS}

Many thanks to Alice and Gordy for letting me go skiing while they completed this manuscript. This work supported in part by the U.S. Department of Energy. 